\title{
The Wisdom of Being Wise A Brief Introduction to Computational Wisdom
}

\author{
Stephen Marsh ${ }^{1}$, Mark Dibben ${ }^{2}$, and Natasha Dwyer ${ }^{3}$ \\ 1 University of Ontario Institute of Technology, Canada. stephen.marsh@uoit.ca \\ 2 University of Tasmania, Australia. Mark. Dibben@utas.edu.au \\ 3 University of Victoria, Australia. Natasha.Dwyer@vu.edu.au
}

\begin{abstract}
This paper explores how the very human notion of Wisdom can be incorporated in the different behvaiour and ultimately reasonings of our computational systems. In particular, it extends and combines previous work in the areas of Computational Trust, Socially Adept Technologies, Device Comfort and the more recent notion of Slow Computing that was teased out at a recent Dagstuhl seminar. A brief exposition of Wisdom, its place in autonomous sociotechnical systems, and pointers to how we can make it work are provided. Further work is explored.
\end{abstract}

\section{On Being Wise, and What it Might Mean for Computers}

the only real wisdom is knowing you know nothing.

Socrates $^{4}$

As humans, we value wisdom. It provides, in those who possess it, a knowledge of how things should be done, how life should be lived - either to the full or in some way that has less impact on, or is more in touch with, the world and society around it. Its gift is the ability to adapt to new, unforeseen, unexpected happenings gracefully, putting into practice experience in order to manage that which has not been encountered before.

Computers and computational systems do not (presently) possess wisdom. It is an unerringly 'natural' phenomenon. ${ }^{5}$ However, we believe that there may be something to be gained from its study, and ultimately the ability to incorporate the behaviours and reasoning into (at least semi-) autonomous computational systems can bring benefit.

This short paper begins the exploration of wisdom and suggests ways in which Computational Wisdom might be achieved. It begins with an exploration of wisdom in the natural world (which includes people), and searches for common traits. It then delves into how these traits can be identified and aimed for in our computational systems, before beginning

\footnotetext{
${ }^{4}$ A possibly equally old saying has it that Wisdom is knowledge that you gain immediately after you need it. .

${ }^{5}$ While there may be some debate, for which this is almost certainly not the right venue, there appears to be evidence that suggests that other animals than humans may possess it [9]
} 
an exploration of a possible framework for what we have come to call Computational Wisdom, and how it relates to previous work. Finally, and perhaps most importantly, it lays out a set of future goals that we can work towards in the search for truly wise, and as a result resilient and people-focused, computational systems.

As a brief aside, this paper may appear highly conjectural, at least at the outset. We make no apologies for this: it's an unusual topic to think of in computational terms, and the very few examples that have done so (see for example [22]) appear to have done so more from the point of view of understanding the human mind than the uses of the concept. Many more useful expositions can be found in the management sciences, for wisdom is very much a tool and a trait of success $[19,18]$. To that end, just as in all our previous work, this is a multidisciplinary journey.

\section{On What Has Come Before}

This is not the first time 'Wisdom' has been evoked in the computational sphere, and it's certainly not the first time wisdom has been studied in the human, psychologically, philosophically, and in religious studies, to name the three more relevant areas of research and discourse. In this section, we discuss the differences between the former and our positions, whilst the next section discusses the latter.

There are probably three main areas where wisdom has been discussed in the computational. The first is in Computational Epistemology [22], the study of knowledge as it relates to and is used by computational systems a root, in one direction, and a branch, looked at in another, of cognitive science. The second is in Harel et al's proposal for Wise Computing [6], the development of systems where the artificial system is capable of being a 'tier 1' member of the designer's club of systems. The third, related and most probably most developed, is the Artificial Sapience [17] movement that sparked in the middle of the first decade of this century, and was focused on knowledge intensive multi-agent systems. Each of these thrusts has something to teach us, but each is ultimately a thrust that attempts to isolate 'wisdom' for explicit use in artificial systems. Our own work acknowledges the place of this, but specifically requires that Wisdom in the Computational sense is a system of systems involving both artificial and 'natural' (or human) - the trick, then, is to know where one wisdom begins and another ends (since wisdom appears to be contextual too [4]).

As well, computing culture has explored one notion of wisdom in the form of the 'wisdom of the crowds' (WOC) movement. However, the type of wisdom espoused by WOC is not what we are referring to in this paper and we wish to differentiate our conceptualisation from common notions of the WOC. Our understanding of wisdom involves a subtle handling of context. In contrast, WOC refers to a practice of gathering the input from a large amount of users who can bring a wide range of expertise, experience and aggregation methods to a scenario [8]. Applications of the WOC include using a wide range of inputs to filter internet content and improve the results of a search engine [20]. While we acknowledge 
that the WOC method can harness a large number of perspectives, it is more a form of collective intelligence does not offer the experience we refer to as 'wisdom'. From a cynical perspective, WOC is simply a way to solve an issue cheaply [24]. Wisdom precedes problem solving and is a form of knowledge that can help reconceptualise a problem situation, including processes such as problem finding and problem making.

Our work differs in some fundamental ways from the previous examinations of wisdom in the artificial. It is not a study of computational epistemology [22], which seeks to explore the means by which we may build truly wise computers, more it is the application of principles of wisdom to the systems that may exist. The difference is, in the spirit of Mark Prensky (see for instance [21]), that true wisdom comes from acknowledging that the answers are not always known, and that the synthesis of ideas (such as computational epistemology seeks in its fulfillment [22]) requires many different inputs, and finally that some of these inputs at the very least are human. Computational Wisdom is a system of systems that is able to understand the value of each part of the system and harness each to their full potential.

On a related note, it's about, but more than, sapience in the concept of Artificial Sapience. Whilst wisdom and sapience are seen as synonymous, particularly in the Western sphere, we see them as different. Indeed, as [26] notes, Western concepts of wisdom are inherently cognitive, whereas the Eastern concept is both cognitive and affective. In our other work to date, and in our concept of Computational Wisdom, we acknowledge the affective as a very important aspect of the whole. Knowledge and cognition (as in the Berlin Wisdom framework [1], for instance) are vital, as is metaknowledge, but the relationship between observer, actor, 'user' (in the sense of a person using' a computer), system and environment are the key, as can be seen in our Device Comfort work, for instance [15]. Wisdom has also been applied in educational settings, and in Suarez's thesis [25] we find the concept of Wisdom "by design", which discusses the notion of artificial wisdom briefly by examining how to design complex social systems that embody wisdom. The work is interesting in that it espouses several principles of wisdom-based design, but its focus on complex societal structures (such as the educational system) means that it is somewhat removed from a computational wisdom as a goal.

Although not a subject for mainstream social science research until the last few decades, the search for an understanding of wisdom has nevertheless seen some important advances. The next section provides an overview of some of the main aspects if this work in the social.

\section{On What it Does Mean for People}

Wisdom is not an unstudied concept, particularly in philosophy, psychology and religion. It is in particular to these disciplines we must turn to more fully understand the phenomenon. Additionally, as we noted above, the cultural components of wisdom are some things we cannot ignore in our examinations [26, 27].

Wisdom is sought by individuals as means to cope [3]. Similar to notions of trust, where an individual seeks to place their faith in someone 
or something, wisdom works as a guiding light. Wisdom is not either completely rational or irrational and is a phenomenon that is difficult to explain [3]. It is a 'big picture' viewpoint that takes into account a broad perspective that values longer timeframes. As Hoefstede argues [7], Western cultures tend not to be orientated towards longer-term conceptualisations of a situation. The emphasis is more on short-term returns. This may explain why notions of wisdom tend to be associated with cultures outside of the West, such as Asian cultures that are sometimes exoticised by the West.

\section{Principles of Wisdom and Their Computational Reflections}

We can begin to see a structure here. Wisdom has certain principles (or behavioural and reasoning patterns) associated with it. As in previous work $[13,15,11]$ we believe that it is possible to isolate these principles and, furthermore, use them in computational settings. In this section of the paper, we begin the former. The following section begins the rather more detailed work required for the latter.

We have been asked, why these particular principles and not others? The simple answer is that these are the beginnings of what we think are relevant. A more complex answer is: we chose these because they provide building blocks, and sensible starting points for researchers considering Computational Wisdom per se. That they are arbitrary is not in question, but we hope they provide food for thought nevertheless.

\section{Principles}

Wisdom works in the user's interests. A we have already noted in [12, $11]$, computing is about and for people. Part of this 'equation' comes with the understanding that, if there is a problem, the solution is not to make life more difficult for people.

Computational Wisdom creates calmness for the user. Rather than subject to corporate interests and 'rational' computations that ignore the richness of human life, the system is working in the user's interests. Nuanced contexts are embraced and users are guided through the messy and contradictory demands of everyday life, keeping a focus on the values that really matter.

Wisdom is Slow. The creation of ever more complex autonomous systems can be seen as, and indeed in many instances is, beneficial. However, as we have argued before (see for instance [11]), there are instances where this is not the case. As we note in [10], slowing the system down to a human level when an 'edge' case arises, where systems do not know the 'answer', can bring benefits, from greater human understanding to more correct decisions (not to mention reduced liability and an ethical stance that is defensible in real ways). It has been pointed out that slowness need not indeed be a human-centric phenomenon for wisdom. We concur: a slowing down of processing or relaxing of time constraints, to allow for 
more consideration or simply reflection on the part of a wise system is, we believe, a vital aspect of how future systems, in the right contexts, can and should behave. Since we also believe that all systems 'touch' people at some point, this can only benefit the human both 'in' and 'out' of the loop.

Computational Wisdom is Multi-Faceted. As [25] notes, there are different aspects of wisdom that can be designed for, including time-sensitivity, balance (between 'vision' and 'action') and practicality. From our point of view, this relates to the idea of human-centric systems, where systems should 'think' before acting. But the idea of a multi-faceted approach is worth exploring: in this short paper we will not be able to bring all of the facets of wisdom to the fore, and make this a priority for future work.

Wisdom is Adaptive. We have noted above the contextual nature of wisdom. It is true that different circumstances require different actions, but further, Computational Wisdom requires that ostensibly the same circumstance may require different actions (for instance, when something new has been learnt by the system as a result of prior actions).

\section{How Can We Engage Wisdom: Thoughts on Computational Wisdom}

Computational Wisdom is seen here as a system of systems, where the technology augments the human in one direction, whilst being augmented by the human in another, and finally where different technological systems, such as the computational trust $[13,16,14]$ comfort $[15,5]$ slow computing [10] and socially adept or intelligence technological systems [2] we and others have already postulated and built, build what wisdom is possible between them.

There are, we conjecture, two main questions to answer on the journey to this system of wisdom. The first is when it should engage or be engaged, whilst the second is what form of engagement is to be exhibited. ${ }^{6}$

Some of the ideas from the social sciences, concerning what can be said to make up a wise person are difficult to make manifest in the AI realm $[18,19]$. These might include: notions of courage and bravery or even perhaps the ability to engage in open productive dialogue; the capacity to appropriately criticise and recognise the need for professional detachment; as well as the capacity to contain one's emotions in the face of negative feedback or behaviour; emotional empathy; and a well-rounded curiosity for life in general. However, it seems to us that a whole host of others might be useful in integrating complex computational systems with the capacity to act wisely. For example, it is already well understood that trust can be instigated in such systems for the purpose of building agency. In addition, the very nature of the highly complex systems we are dealing with require the ability for $\mathrm{AI}$ agents to make clear decisions (qua judgements) in the face of paradoxes and ambiguity; the formalism for this are an inherent part of information systems.

\footnotetext{
${ }^{6}$ Of course, the answers to these questions contain an element of wisdom in and of themselves. .
} 
It seems to us, then, that wisdom within computing insofar as the capacity to deal with uncertainty through statistical solutions to unknowns brings us close to something of what we mean by wisdom within computing systems, much as Sevilla notes [23]. What more is needed? First, some aspect of what we might anthropocentrically call 'humility', but in this setting can be seen as computational systems that are capable of taking their own limitations into account, and also of referring to other parts of the system for information when necessary. In addition, since complex systems are already suffused with algorithms for making predictive decisions of desired content, based on learnt knowledge of (e.g.) a user's browsing behaviour, so it is not a large step to refine these types of algorithms to allow agents to recognise when it is not appropriate to share knowledge with other agents.

Another aspect that McKenna and Rooney suggest as being fundamental to wise leaders, introspection, can also be seen in complex computational systems. That is, they can be configured to recognise errors, seek out reasons for them and use that reasoning to inform future decisions. This, in turn, can be extended to the capacity question established norms, systems, processes and procedures. In addition, the capacity for machine learning provides the means for AI agents to behave as if they were prudent. That is, act in the right way, based on formal logic and reasoning, modified perhaps by algorithms intended to convey human values such as trustworthiness and an understanding of importance and risk.

One aspect of wisdom that may be more difficult to apply concerns the way in which wise judgment takes into account intuition, political acumen, capacity for tact, subtlety and shrewdness, and also may be based on recognition of a higher purpose or the common good. That said, were these pre-determined as part of the complex system's purpose and the necessary algorithms constructed to focus on that higher end, even this aspect of wisdom may be incorporated. The learnt component of decision making may also enable the system to accommodate wrong answers (i.e. setbacks or disappointments) and behaving as if they were being more cautious in situations entailing risk.

\section{The Future, Perhaps}

There is, we believe, promise in the short study of wisdom that we have heretofore made. It is a powerful notion with acknowledged strengths. Moreover, it lends itself to a set of principles that we can isolate and begin to work with. Finally, it has within it enough hooks and identifiable requirements to be able to construct a framework around it that allows computational systems to recognise the need for it, engage its principles, and formulate behaviours that exhibit some at least of its strengths. In short, it is an ideal object of study.

A short paper such as this is a poor exposition of a powerful notion, but its aim is to provide both an inkling of the potential as well as ideas as to how this can be studied and worked towards. We hope that the previous sections have gone some way toward the former. Our future work will take this further, and provide insights into the latter. 


\subsection{On Future Work}

There have been some attempts to define a research agenda to explore wisdom in the computational, most promisingly that of [23] and the notion of Artificial Sapience [17]. In most cases, the goal is an intelligent system that behaves wisely, for some definition of wise. As we have already noted above, we see the limitations here, that such systems are not oriented toward to very people that would work alongside them, and this is a failing.

To address this, we explicitly acknowledge the human in the system as an equal (if not, in this setting, superior) partner. The human is where the system can learn from, and fall back to, in difficult situations, for example. And so, in any study of computational Wisdom, we must start with the human element. Our work is currently taking us in the direction of user engagement with complex sociotechnical systems, automation and information, and this is no exception. Our next step for Computational Wisdom $\mathrm{s}$ indeed to set goals in collaboration with human users to ascertain the best uses and directions for 'wise' systems to take, what they might look like, and how they might express themselves to their human partners.

\subsection{A Conclusion}

Notwithstanding certain emotional attributes of wisdom may be perhaps beyond the capacity of complex computational systems, many aspects of wisdom can be modeled and integrated into their decision making processes. Our thesis is this: Wisdom is a difficult multi-faceted construct to grasp (and indeed, to attain, hence its value!). It's certainly worth studying computationally, and importantly, as we have previously discovered with trust, it might indeed be possible to create computational systems that act as if they are wise. This would be a rich extension to systems modeled to act as if they were trusting and trustworthy, because it would bring a wider range of factors to bear upon the system and its outputs. In order to get there, we need to comprehensively study and include the many different spheres of study that have touched on the concept.

Wisdom is powerful, well respected, and in all systems, a goal: we strive to be wise, to behave wisely, and to be seen as wise. This paper argues that the goal of wisdom is something that we can also work towards for the artificial systems that we create to take their place in our societies. Such systems can be more focused on the 'wise' thing to do in any given circumstance, and would naturally include the traits of critical thought, the identification of when to engage wisdom and why (or why not), a focus on the well-being of the society and the people around them, as well as the other systems that exist, and, we conjecture, a calmer and slower approach to reasoning that engages rather than alienates the very people that the systems serve.

It is a long road. This is one of the first steps. 


\section{Acknowledgments}

We thank the anonymous reviewers for their erudite comments and thoughts, which have helped make our thoughts more concrete. The first author gratefully acknowledges the support of the Natural Sciences and Engineering Research Council of Canada under the Discovery Grants Program.

\section{References}

1. P. Baltes and U. Staudinger. Wisdom: A metaheuristic (pragmatic) to orchestrate mind and virtue toward excellence. American Pscyhologist, pages 122-136, January 2000.

2. Kerstin Dautenhahn, Bond Alan H, Lola Canamero, and Bruce Edmonds, editors. Socially Intelligent Agents: Creating Relationships with Computers and Robots. Kluwer Academic Publishers, 2002.

3. K. Gehrman. Absorbed coping and practical wisdom. The Journal of Value Inquiry, pages 1-20, 2015.

4. S. R. Grimm. Wisdom. Australasian Journal of Philosophy, 93(1):139-154, 2015.

5. Jingjing Guo, Christian D. Jensen, and Jianfeng Ma. Continuous context-aware device comfort evaluation method. In Christian Damsgaard Jensen, Stephen Marsh, Theo Dimitrakos, and Yuko Murayama, editors, Proceedings of the 9th IFIP WG 11.11 International Conference on Trust Management IX (IFIPTM 2015), pages 203211. Springer AICT, 2015.

6. D. Harel, G. Katz, R. Marelly, and A. Marron. Wise computing: Towards endowing system development with true wisdom. arXiv preprint arXiv:1501.05924, 2015.

7. G. Hoefstede. Cultures and organizations: Software of the mind. McGraw-Hill, New York, 1997.

8. M. Hosseini, J. Moore, M. Almaliki, A. Shahri, K. Phalp, and R. Ali. Wisdom of the crowd within enterprises: Practices and challenges. Computer Networks, 90:121-132, 2015.

9. Jon Lieff. Wise animals: Animal studies need to be in natural settings, not lab. http://jonlieffmd.com/blog/wise-animals-2 viewed February 20th, 2016, 2012.

10. S. Marsh. All human values are system values. Abstract and Talk given at Dagstuhl Seminar on Social Aspects of Self-Organizing Systems, November 23, 2015.

11. S. Marsh, A. Basu, and N. Dwyer. Rendering unto caesar the things that are caesar's: Complex trust and human understanding. In Proceedings IFIPTM 2012: Trust Management VI, Surat India, pages 191-200. Springer AICT, 2012.

12. S. Marsh, N. Dwyer, A. Basu, K. El-Khatib, T. Storer, B. Esfandiari, K. Renaud, and M. Bicacki. Foreground trust as a security paradigm: Turning users into strong links. In A. Kayem and C. Meinel, editors, Information Security in Diverse Environments. IGI Global, 2014. 
13. Stephen Marsh. Formalising Trust as a Computational Concept. $\mathrm{PhD}$ thesis, University of Stirling, 1994. http://www.stephenmarsh.ca/pubs/Trust/PhD/Trust.pdf.

14. Stephen Marsh and Pamela Briggs. Examining trust, forgiveness and regret as computational concepts. In Jennifer Golbeck, editor, Computing with Social Trust, Human Computer Interaction Series, chapter 2, pages 9-44. Springer, 2009.

15. Stephen Marsh, Pamela Briggs, Khalil El-Khatib, Babak Esfandiari, and John A Stewart. Defining and investigating device comfort. Information and Media Technologies, 6(3):914-935, 2011.

16. Stephen Marsh and Mark R. Dibben. Trust, untrust, distrust and mistrust - an exploration of the dark(er) side. In Peter Herrmann, Valerie Issarny, and Simon Shiu, editors, Trust Management: Proceedings of iTrust 2005. Springer Verlag, Lecture Notes in Computer Science, LNCS 3477, 2005.

17. R. V. Mayorga and L. I. Perlovsky. Toward Artificial Sapience: Principles and Methods for Wise Systems. Springer, 2007.

18. Bernard McKenna. The multi-dimensional character of wisdom. In Mike J. Thompson and David Bevan, editors, Wise Management in Organisational Complexity, chapter 2, pages 13-33. Palgrave MacMillan, 2013.

19. Bernard McKenna and David Rooney. Wise leadership and the capacity for ontological acuity. Management Communication Quarterly, 21:537-546, 2008.

20. C. Padoa, D. Schneider, J. Moreira de Souza, and P. J. Medeiros. Investigating social curation websites: A crowd computing perspective. In Computer Supported Cooperative Work in Design (CSCWD), 2015 IEEE 19th International Conference on, pages 253-258. IEEE, 2015.

21. Mark Prensky. Brain Gain: Technology and the Quest for Digital Wisdom. Palgrave MacMillan, 2012.

22. N. Rugai. Computational Epistemology: From Reality to Wisdom. Lulu Press, 2012.

23. David Casacuberta Sevilla. The quest for artificial wisdom. AI and Society, 28:199-207, 2011.

24. E. Simperl. How to use crowdsourcing effectively: Guidelines and examples. Liber Quarterly, 25(1), 2015.

25. Juan Francisco Suarez. Wise by Design: A Wisdom-Based Framework for Innovation and Organizational Design and its Potential Application in the Future of Higher Education. PhD thesis, Antioch University, Dissertations \& Theses. Paper 131. http://aura.antioch.edu/etds/131, 2014.

26. M. Takahashi and P. Bordia. The concept of wisdom: A crosscultural comparison. International Journal of Psychology, 35(1):1-9, 2000 .

27. Masami Takahashi. Toward a culturally inclusive understanding of wisdom: Historical roots in the east and west. International Journal of Aging and Human Development, 51(3):217-230, 2000. 